\title{
Oscillating reactions at metal electrodes in solid electrolytes
}

\author{
Jürgen Janek* \\ Institut für Physikalische Chemie und Elektrochemie und Sonderforschungsbereich 173, Universität Hannover, Callinstrasse 3-3A, \\ 30167 Hannover, Germany
}

\begin{abstract}
The transfer of matter and charge across interfaces between two solids is generally related to processes of defect relaxation in the regions near the interface. A transfer rate which exceeds the rate of defect relaxation may lead to degradation of the interface, causing a feedback effect for the transfer itself. As a consequence, non-linear phenomena (dissipative structures) like periodic oscillations of the interfacial properties can occur under conditions far from equilibrium. Experimental examples are discussed.
\end{abstract}

Keywords: Dissipative structures; Electrochemical oscillations; Interfaces; Ionic conductors; Point defects

Materials: AgI; $\mathrm{CoO}$

\section{Introduction}

Oscillatory (electro-)chemical reactions are well known examples for the existence of dissipative structures. They are generally based on the coupling of diffusive and reactive processes including nonlinear kinetic terms [1]. Interfaces between two solids, which show atomic mobility due to the existence of mobile lattice defects, are places where both transport and reaction are necessary steps in order to allow continuous matter transfer from one solid to the other. Thus, at least from an intuitive point of view, solid-solid interfaces provide necessary ingredients for the occurrence of dissipative structures. From a mere phenomenological point of view, equivalent situations are to be found at electrode interfaces in liquid electrolytes. And as it is

\footnotetext{
${ }^{*}$ Corresponding author. Tel.: +49 511 762-5298; fax: + 49511 762-4009; e-mail: janek@mbox.pci.uni-hannover.de
}

well known since the pioneering works of Bonhoeffer [2] and Franck [3], electrochemical oscillations can indeed be observed during charge transfer across electrodes in liquid electrolytes (see Ref. [4] for a survey). The potential dependent formation (and dissolution) of electrically isolating surface layers (e.g., oxides) is often an important prerequisite for the occurrence of these oscillations. The existence of comparable phenomena at interfaces of solid electrolytes is demonstrated in the present work. Analogies and differences to the oscillations at electrodes in liquid electrolytes are briefly discussed.

The basic kinetic situation during the transfer of matter across a solid-solid interface can be summarized following the ideas of Schmalzried [5], thus, the exchange of matter and charge between two solid phases is regarded as a combination of heterogeneous and homogeneous defect reactions. For example, regarding the exchange of cations $\mathrm{Me}^{+}$between two cation-conductors $\alpha$ and $\beta$, it is not sufficient for 
a complete understanding to formulate this process simply as $\mathrm{Me}^{+}(\alpha) \rightleftharpoons \mathrm{Me}^{+}(\beta)$. Rather one has to take the defect structures of both phases into account, in order to provide a suitable kinetic description. If we assume that both solid phases $\alpha$ and $\beta$ show Frenkel disorder in their cation sublattices, different situations arise whether an ion moves from the regular or the interstitial sublattice in $(\alpha)$ to the regular or interstitial sublattice in ( $\beta$ ). A thorough analysis and formal treatment of this kinetic problem at an interface has been given by Schmalzried ([5], p. 247 ), leading to the definition of a relaxation overvoltage which bears the influence of a slow homogeneous defect equilibrium on the interfacial transport.

Regarding ion transfer across solid-solid interfaces, another basic kinetic situation is the exchange of metal atoms (ions) between a parent metal and a corresponding cation conductor [6] as it is depicted schematically in Fig. 1. In an idealized picture, the anodic electrode process comprises the consumption of a cation vacancy $\mathrm{V}_{\mathrm{Me}}^{\prime}(\mathrm{MeX})$ or the injection of a cation interstitial $\mathrm{Me}_{\mathrm{i}}(\mathrm{MeX})$ on the side of the solid electrolyte. Since the mobility of the ionic defects in solid electrolytes is generally high enough, the further homogeneous transport of these defects will not cause any serious kinetic hindrance to the overall process. In addition to the processes in the solid electrolyte, a metal vacancy $\mathrm{V}_{\mathrm{Me}}(\mathrm{Me})$ is created on

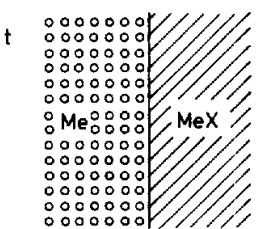

$$
\Leftrightarrow\left|V_{p G}^{a}\right| \propto i
$$

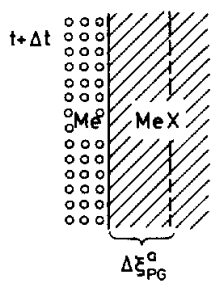

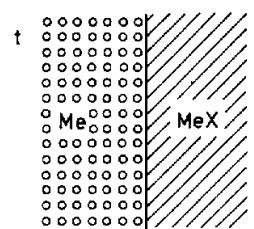

\& $\left|V_{P G}^{b}\right|<\left|V_{P G}^{a}\right|$

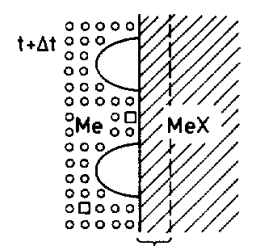

$\left|\Delta \xi_{\mathrm{PG}}^{\mathrm{b}}\right|<\left|\Delta \xi_{\mathrm{PG}}^{\mathrm{c}}\right|$
Fig. 1. Schematic representation of the moving interface (interface velocity $V_{\mathrm{PG}}$ ) during ion transfer across an interface (space coordinate $\xi$ ) between a parent metal and a cationic conductor: (a) without and (b) with pore formation. the metal surface, which can be expressed by the simple electrode reaction:

$\mathrm{Me}_{\mathrm{Me}}(\mathrm{Me})=\mathrm{Me}_{\mathrm{i}}(\mathrm{MeX})+\mathrm{e}^{\prime}(\mathrm{Me})+\mathrm{V}_{\mathrm{Me}}(\mathrm{Me})$.

In order to maintain the original interface structure, the metal vacancies have to migrate away from the surface or have to be annihilated at places of repeatable growth. As our findings for the $\mathrm{Ag} \mid \alpha-\mathrm{AgI}$ interface show, Eq. (1) is definitely a strong oversimplification of the real electrode process and is not sufficient to understand its kinetics completely.

Another basic kinetic situation is realized during the anodic dissolution of a cation-conducting solid electrolyte (in the present case we specify the general case to a semi-conducting oxide of the type $\mathrm{A}_{\mathrm{I}-\delta} \mathrm{O}$ ) at an inert metal electrode, see Fig. 2b. The electrode reaction leads to the annihilation of negatively charged cation vacancies, $V_{A}^{\prime \prime}$, which migrate to the anode in the electric field (counter-diffusion of cations $\mathrm{A}_{\mathrm{A}}^{\mathrm{x}}$ ), and to the oxidation of oxygen ions which leave the crystal then as molecular oxygen:
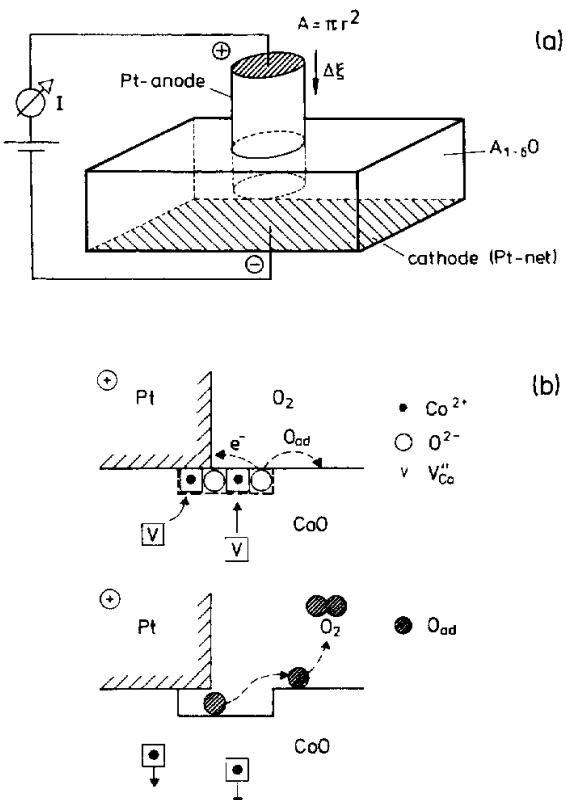

(b)

Fig. 2. Electrolysis of a semiconducting oxide $\mathrm{A}_{1-\delta} \mathrm{O}$ at an inert metal (point) electrode. (a) Schematic representation of the experiment, (b) anodic electrode process and formation of surface defects. 
$\mathrm{AO}+\mathrm{V}_{\mathrm{A}}^{\prime \prime}=\frac{1}{2} \mathrm{O}_{2}+\mathrm{A}_{\mathrm{A}}^{\mathrm{x}}+2 \mathrm{e}^{\prime}+\mathrm{V}_{\mathrm{AO}}$ (surf).

As in the case of the parent metal electrode $\mathrm{Ag} \mid \alpha-\mathrm{AgI}$, the anodic electrode reaction leads to the creation of vacant lattice sites at the interface. In the case of $\mathrm{Pt} \mid \mathrm{CoO}$, these vacant sites can be identified as missing lattice molecules $\mathrm{V}_{\mathrm{AO}}$ (surf) of $\mathrm{CoO}$.

\section{Experimental observations}

Both periodic and irregular oscillations of the interfacial overvoltage have been observed at the $\mathrm{Ag} \mid \alpha$-AgI interface under galvanostatic conditions [6]. Their appearance depends on the current density, the temperature and most critically on the applied mechanical pressure (see Ref. [7]). It has to be noticed that it is almost impossible to reproduce a certain oscillation mode, i.e. different cells show different oscillation modes even under identical experimental conditions. Besides the electrochemical experiments, the interfaces have been studied by (electron and opticai) microscopy after the experiments and by in-situ dilatometric [8] and in-situ optical measurements (reflectivity of the electrode) in order to obtain further experimental information on the origin of the oscillations. In conclusion, the alternating production and annihilation of small pores (accumulated metal vacancies) and the related changes of the effective electrode area are responsible for the oscillation of the overvoltage. Strong experimental support for this conclusion is provided by the in-situ dilatometric studies.

In Fig. 3, a typical time series of galvanostatic voltage oscillations at an $\alpha-\mathrm{Ag} \mid \mathrm{AgI}$-interface is depicted (see figure for experimental conditions). An insert shows a more time-resolved part of the time series. Most of the characteristic features of the oscillatory kinetics are all found within this single time series: (a) very often, the oscillation mode changes continuously during a single experiment without a detectable change of experimental parameters ('mixed mode' oscillations), (b) typically, the oscillations can be characterized due to the shape of their peaks as 'relaxation oscillations' with a slow rise of the voltage and a quick drop of the voltage back to a very low voltage, (c) generally, the voltage remains considerably smaller than the thermody-

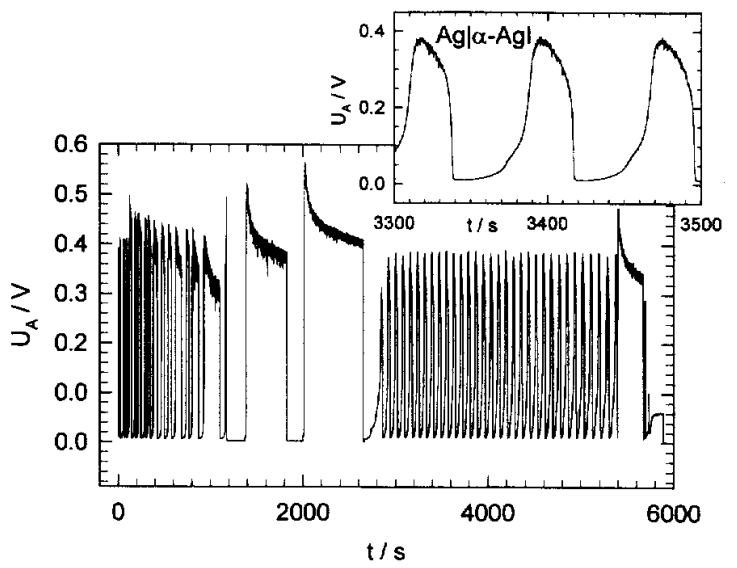

Fig. 3. Electrochemical oscillations $\left(T=300^{\circ} \mathrm{C}, i=1.56 \mathrm{~mA} / \mathrm{cm}^{2}\right.$, $p \simeq 1$ bar) at the anodic interface $\mathrm{Ag} \mid \alpha-\mathrm{AgI}$ : voltage $U_{\mathrm{A}}$ across the interface during galvanostatic dissolution of silver.

namic decomposition voltage of AgI, (d) besides time series which appear chaotic, very regular monoperiodic oscillations as in Fig. 3 can also be observed. More experimental details can be found in Refs. [6,8].

Both periodic and irregular galvanostatic voltage oscillations have also been observed at point electrodes of the type $\mathrm{Pt} / \mathrm{CoO}$. Again, the current density, the temperature and the mechanical pressure are important parameters which control the appearance of the oscillations. Additionally, the oxygen partial pressure influences the oscillation modes by controlling the concentration of cation vacancies in $\mathrm{Co}_{1-\delta} \mathrm{O}$ (heterogeneous equilibrium: $\frac{1}{2} \mathrm{O}_{2}=\mathrm{V}_{\mathrm{Co}}^{\prime}+$ $\mathrm{O}_{\mathrm{O}}^{\mathrm{x}}+\mathrm{h}^{*}$ ). It has to be noticed that the experimental situation is different compared to the $\mathrm{Ag} \mid \alpha-\mathrm{AgI}$ interface at which only ions are exchanged. $\mathrm{Co}_{1-\delta} \mathrm{O}$ is a semiconductor with a very small ionic conductivity. The ionic transference number is of the order $t_{\text {ion }} \simeq 10^{-4}$ at the experimental conditions, and thus, a total current of $1 \mathrm{~mA}$ leads to an ionic current of only $0.1 \mu \mathrm{A}$. Thus, the observed oscillations are caused by the degradation of the interface due to migrating ions, but this degradation is probed by the interface resistance for electronic charge carriers.

Different point electrodes with both spherical or cylindrical shapes (diameter $300 \mu \mathrm{m}$ ) have been used, showing no serious difference in behaviour. Since the electrodes are slowly sinking into the oxide 
single crystals during an experiment, the electrode area is generally increasing during the experiments. Thus, under galvanostatic conditions, the current density at the interface decreases continuously. Assuming an electrode area of $100 \mu \mathrm{m}^{2}$, a total current of $10 \mathrm{~mA}$ leads to an ionic current density of approximately $10 \mathrm{~mA} / \mathrm{cm}^{2}$. If one further assumes that the interface area shows a maximum during the voltage minima between two voltage peaks, one can apply the theory of constriction resistances $\left(R_{\mathrm{AO}}=\right.$ $\left(2 \pi a \cdot \sigma_{\mathrm{AO}}\right)^{-1}$ for a semi-spherical point electrode, with $a$ denoting the radius of the semi-sphere and with $\sigma_{\mathrm{AO}}$ denoting the electric conductivity of the oxide [9]) to determine the conductivity of the electrolyte. Since the electronic conductivity of $\mathrm{Co}_{1-\delta} \mathrm{O}$ is well known, it is possible in the reverse order to compare the experimental and the theoretical constriction resistance of the point electrode for a given geometry. Not surprising, the constriction resistance which is derived from the voltage minima is considerably higher than the theoretical resistance of the respective ideal point contact (as calculated using geometrical information from SEM pictures, cf. Fig. 4). Thus, the real $\mathrm{Pt} \mid \mathrm{CoO}$ point contact has to be regarded itself as a combination of even

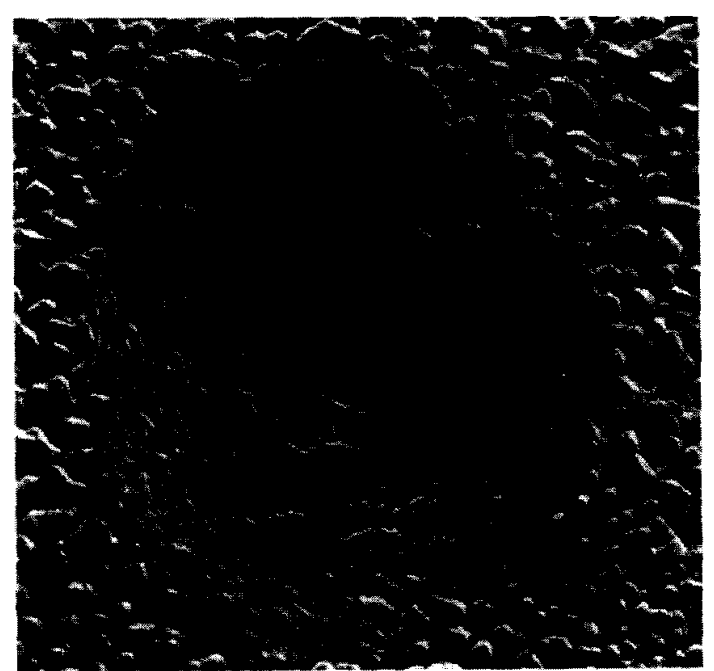

$30 \mu \mathrm{m}$

Fig. 4. Surface of a $\mathrm{CoO}$ single crystal after an electrotransport experiment; the SEM picture shows the electrochemically produced indentation of the semi-spherical platinum anode.

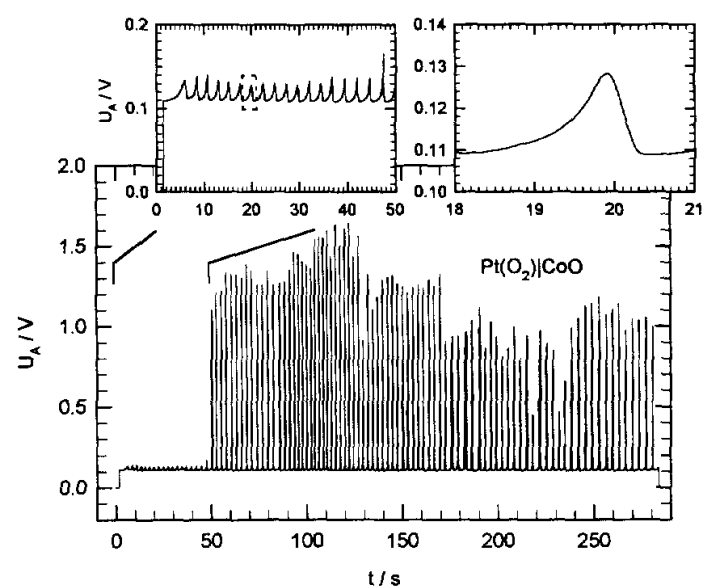

Fig. 5. Electrochemical oscillations at the anodic interface Pt $\mid \mathrm{CoO}$ : voltage $U_{\mathrm{A}}$ across the interface during galvanostatic dissolution of $\mathrm{CoO}\left(T=1100^{\circ} \mathrm{C}, a\left(\mathrm{O}_{2}\right)=0.21, I=8 \mathrm{~mA}\right)$.

smaller point contacts which are a consequence of the morphologically unstable interface.

Regular oscillations were mainly observed during early stages of the experiments, i.e. when the electrode area is still relatively small. In Fig. 5, a typical example is depicted, showing almost periodic oscil-

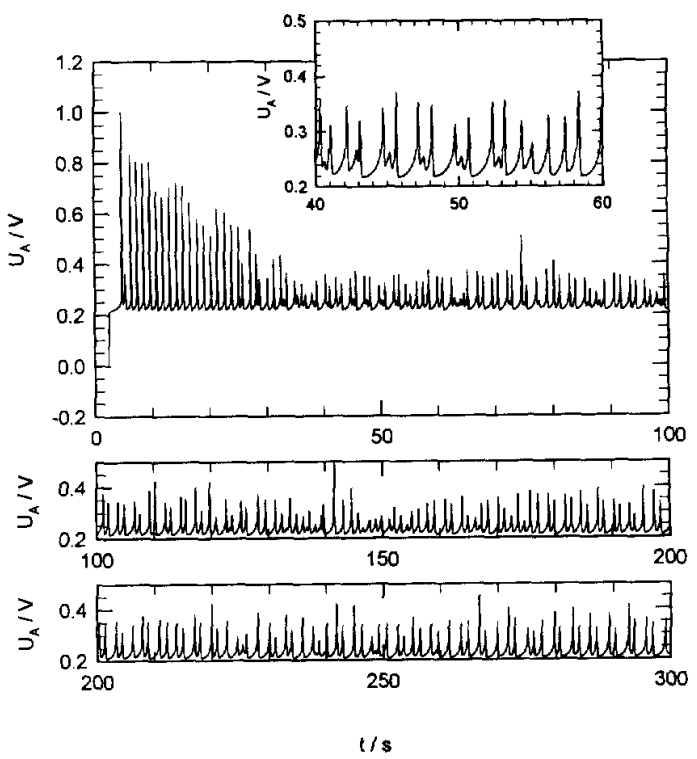

Fig. 6. Electrochemical oscillations at the anodic interface $\mathrm{Pt} / \mathrm{CoO}$ : voltage $U_{\mathrm{A}}$ across the interface during galvanostatic dissolution of $\mathrm{CoO}\left(T=1100^{\circ} \mathrm{C}, a\left(\mathrm{O}_{2}\right)=0.21, I=16 \mathrm{~mA}\right)$. 
lations with a frequency of approximately $0.37 \mathrm{~s}^{-1}$ (see figure for experimental conditions; electrode diameter $300 \mu \mathrm{m}$ ). The two inserted figures with more time-resolved parts of the time series show that the observed voltage peaks are not as sharp as they appear. Thus, the scattering of the amplitude is not an experimental artefact caused by a too slow data acquisition or by a too slow galvanostat, rather it is a characteristic feature of the oscillations (frequency of data sampling: $500 \mathrm{~Hz}$ ). In Fig. 6, a different time series is depicted, showing both regular and irregular oscillatory behaviour.

\section{Discussion and conclusions}

The observed voltage oscillations at moving solidsolid interfaces are examples for the occurrence of dissipative structures in solid systems. Whereas periodic spatial phenomena are frequently observed in the course of solid state reactions, no electrochemical oscillations in solid systems have been reported to date. As for the liquid state, the oscillatory kinetics express the complicated dynamics at the non-equilibrium interfaces. However, differing from the well reproducible oscillatory kinetics of metal dissolution in liquid electrolytes, the metal dissolution in solid electrolytes is much less reproducible in a quantitative manner. And whereas the oscillatory kinetics in liquid electrolytes is generally related to film formation, the origin of the oscillatory kinetics in the present cases is due to the accumulation of lattice defects.

From a chemical point of view the $\mathrm{Ag} \mid \alpha-\mathrm{AgI}$ electrode can be regarded as a very simple interface system. However, it is an incoherent interface from a structural point of view, and it is probably more instructive to regard this interface as a combination of two surfaces rather than an interface. The transfer of charge is only possible by the dissolution of silver and the formation of (surface) lattice defects in (on) the silver metal. The formation of pores at the interface is a definite indication for the influence of surface diffusion and causes a mechanical instability during the transfer. A dilatometric study of the anodic silver dissolution shows that the pore volume oscillates simultaneously with the electrochemical signal. Thus, at least two different microscopic transfer processes counteract during the anodic dissolution: (a) surface diffusion-assisted transfer of silver adatoms leads to the formation of pores at the interface. Iodine which can be present within the pores might accelerate the surface diffusion; (b) direct transfer of silver atoms at contact points reduces the pore volume. In addition, plastic deformation of the solid electrolyte may enhance the annihilation of pore volume and thus cause a mechanical feedback effect.

The Fourier (power) spectrum $P(\omega)$ of the periodic part of the time series in Fig. 3 is depicted in Fig. 7. It shows the typical features of a monoperiodic oscillation with overtones. However, the autocorrela-

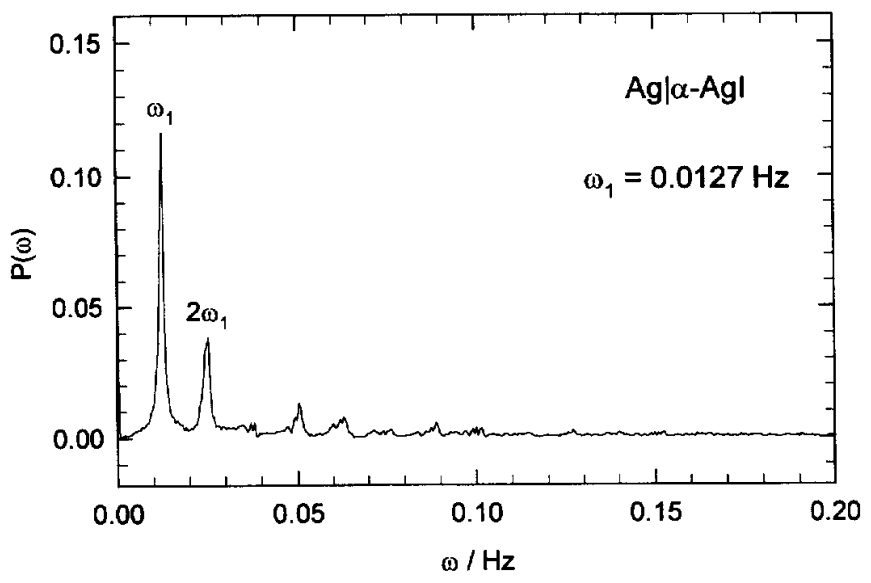

Fig. 7. Fourier (power) spectrum (linear scales) of the time series in Fig. 3; only the part between $t=3000 \mathrm{~s}$ and $t=5400 \mathrm{~s}$ is analysed. 


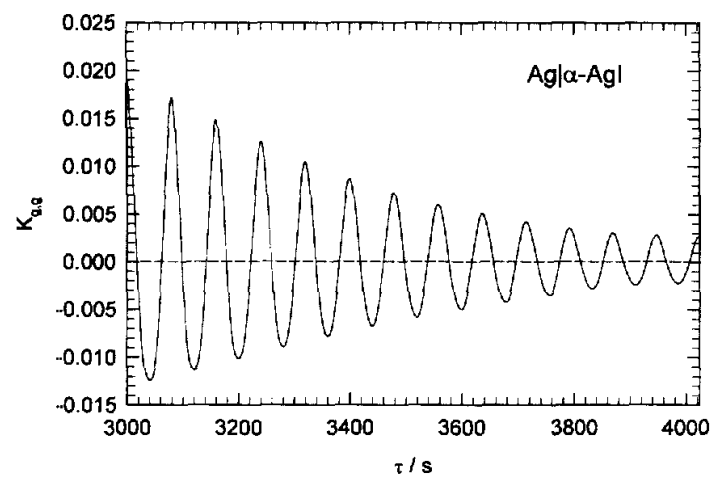

Fig. 8. Autocorrelation function of the time series in Fig. 3; only the part between $t=3000 \mathrm{~s}$ and $t=4000 \mathrm{~s}$ is analysed.

tion function $K_{\mathrm{g}, \mathrm{g}}$ of the same time series is depicted in Fig. 8 and gives important additional information. Whereas a stationary periodic oscillation has a stationary periodic autocorrelation function, the autocorrelation function of the time series in Fig. 3 is a damped periodic oscillation. Since the amplitude of the voltage oscillations remains constant, this can only be caused by a slowly varying frequency of the measured oscillations. Thus, the regular appearing time series does not represent a stationary system. Finally, a comment has to given on the frequency dependence of the power spectrum. In the analysis of electrical noise, a $\omega^{-1}$ dependence of the power is often found (more precisely: $\omega^{-a}$ with $0.8<a<1.2$ ). Recently, this frequency dependence has been interpreted as a consequence of deterministic chaos but still a definite proof is lacking [10]. The power spectra of our oscillations always show an extended $\omega^{-a}$ power dependence with $a \simeq 1$ (see Fig. 9). At the present state, this has to be taken merely as an experimental fact, and a definite proof for the occurrence of deterministic chaos during the electrodissolution of silver in $\alpha$-AgI cannot be given.

Completely different in the underlying chemical reactions but comparable in its phenomenology appears the voltage instability of the $\mathrm{Pt} \mid \mathrm{CoO}$ point electrodes. As for the $\mathrm{Ag} \mid \alpha-\mathrm{AgI}$ electrode it is difficult to observe long time series with a stationary oscillation mode. As is seen in Fig. 5, the oscillations are irregular in respect to the amplitude but almost periodic regarding the frequency. As a consequence, the Fourier (power) spectrum is relatively noisy but

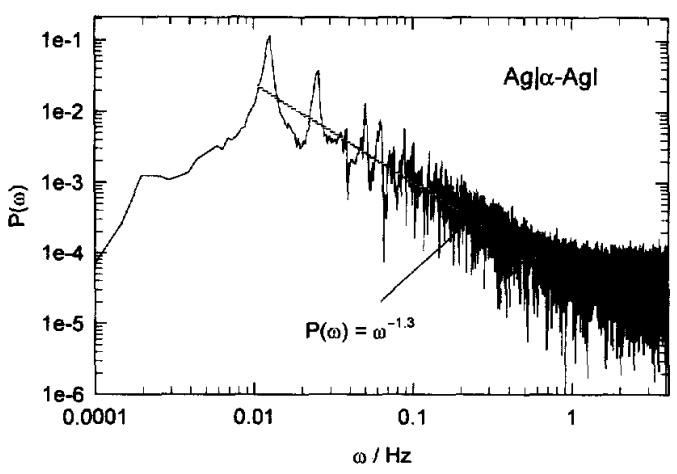

Fig. 9. Logarithmic plot of the Fourier (power) spectrum in Fig. 7.

still a dominating frequency can be identified, see Fig. 10. Again, the logarithmic plot of the power spectrum (Fig. 11) shows a $\omega^{-1}$ dependence of the power.

The application of suitable mathematical techniques for the description and modelling of the observed phenomena is one way to obtain a better understanding $[10,11]$. Thus, a more elaborate analysis of the observed time series might improve our understanding of the underlying dyamics. However, before techniques like the construction of attractors and the calculation of Lyapunov exponents can be applied, stationary time series have to be collected. So far, the observed oscillations are mostly nonstationary and show the characteristics of systems with varying parameters or boundary conditions.

A second route for the better understanding of the oscillatory kinetics may be based on the formulation

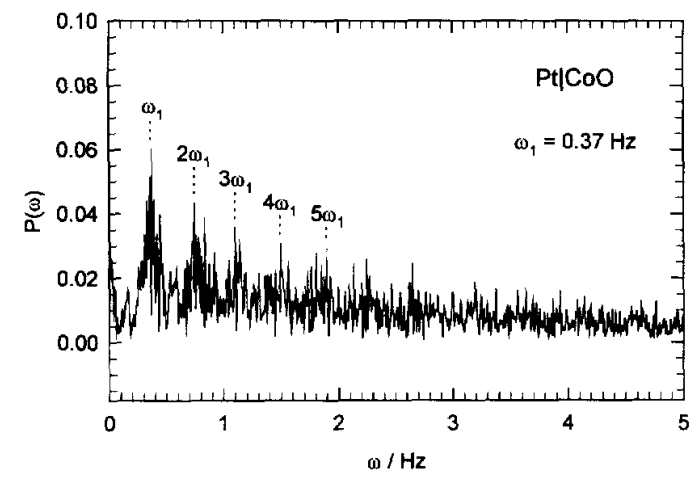

Fig. 10. Fourier (power) spectrum of the complete time series (150000 data points) in Fig. 5. 


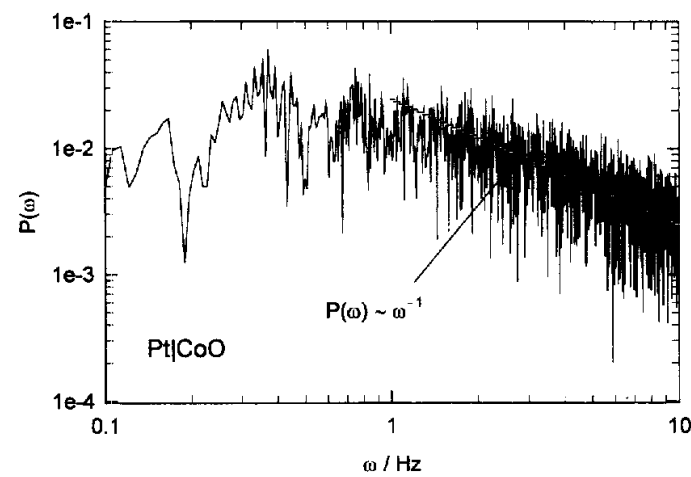

Fig. 11. Logarithmic plot of the Fourier (power) spectrum in Fig. 10.

of physico-chemical models of the interfacial processes and their mathematical expression. The resulting systems of differential equations have to be studied by means of a bifurcation analysis. A systematic application of both mathematical tools for a better understanding is part of present work and will be published elsewhere.

\section{Acknowledgements}

Financial support by the Fonds der Chemischen Industrie (FCI) is gratefully acknowledged.

\section{References}

[1] S.K. Scott, Chemical Chaos, Clarendon Press, Oxford, 1991.

[2] K.F. Bonhoeffer, U.F. Franck, Z. Elektrochem. 55 (1951) 180

[3] U.F. Franck, R. Fitzhugh, Z. Elektrochem. 65 (1961) 156.

[4] See chap. 11 (Electrochemical dissolution reactions) in Ref. [1].

[5] H. Schmalzried, Chemical Kinetics of Solids, Verlag Chemie, Weinheim, 1995.

[6] J. Janek, S. Majoni, Ber. Bunsenges. Phys. Chem. 99 (1995) 14.

[7] J. Janek, S. Majoni, Defect Diff. Forum 129-130 (1996) 243.

[8] S. Majoni, J. Janek, submitted to Ber. Bunsenges. Phys. Chem., 1997.

[9] R. Holm, Electric Contacts: Theory and Applications, Springer-Verlag, Berlin, 1979.

[10] F.W. Schneider, A.F. Münster, Nichtlineare Dynamik in der Chemie, Spektrum Akademischer Verlag, Heidelberg, 1996, p. 95.

[11] T. Kruel, Zeitreihenanalyse nichtlinearer Systeme, Ph.D. Thesis, University of Würzburg, 1992. 